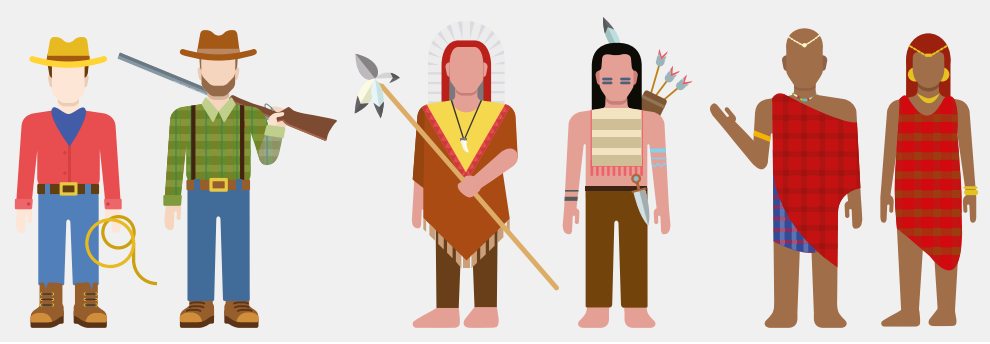

\title{
Nutzen wir die richtigen Waffen?
}

Im Sommer 2015 feierte die American Diabetes Association (ADA) bei ihrer Jahrestagung in Boston/USA ihren 75. Geburtstag. Gegründet im 2. Weltkrieg hatten die Gründerväter das Ziel, auch den Krieg gegen Diabetes zu gewinnen. In seiner Rede kam der aktuelle ADA-Präsident Samuel Dagogo-Jack zu dem Schluss „War on diabetes is challenging, but winnable“! Ist das realistisch?

Seit der Gründung der ADA hat sich die Zahl der Menschen mit Diabetes weltweit vervielfacht. Im Vordergrund steht Typ2-Diabetes, der durch modernen Lebensstil ausgelöst wird. Daher die Frage - um im Bild der US-Kriegsführung zu bleiben welche Waffen im „Krieg gegen Diabetes“ eingesetzt werden? Bei der Analyse der wissenschaftlichen Beiträge der ADA-Tagung 2015 kommt man zu dem Ergebnis, dass ein Großteil der Abstracts pharmakologische Studien sind. Auch der Rundgang durch die Industrieausstellung zeigte, dass die Pharmabranche mit „schwerem Geschütz “ gegen Diabetes vorgeht. In ähnlicher Weise präsentieren sich Medizinproduktefirmen - mit Insulinpumpen bis hin zu Geräten zur kontinuierlichen Blutzuckermessung. Ein weiteres „Waffenarsenal“ besteht aus unzähligen molekularen Forschungsergebnissen. Dort nutzt man erstaunliche Tiermodelle, bei denen u.a. Gene in Hirnarealen oder anderen Zellen ausgeschaltet oder überexprimiert werden. Immer neue Botenstoffe werden entdeckt und deren Bedeutung bei der Entwicklung des Diabetes geprüft. Ziel der Molekular-Analysen ist die Identifikation von Targets für neue Medikamente, z.B. eins, das fehlende Bewegung oder übermäßige Ernährung kompensiert. Von den 2373 präsentierten Abstracts beim ADA 2015 widmeten sich nur 19 dem Thema „Ernährung“ und 33 der Bewegung, also den Faktoren, die die Diabetesepidemie verursachen. Rein numerisch ist das „Waffenarsenal“, das die Ursache des Typ-2-Diabetes bekämpft, mit nur 2\% der Beiträge nicht sehr stark aufgestellt. Die Hintergründe dafür sind vielfältig. Aus der Sicht der Industrie z.B. ist Diabetes ein unglaublich großer Markt. Speziell der Anstieg der Diabetesprävalenz in sich entwickelnden Ländern wie China eröffnet fast unbegrenzte Möglichkeiten der Expansion.

\section{Klinisch-diabetologische Forschung - Fehlanzeige}

Die molekulare Forschung wird mit sehr großem finanziellem Aufwand gefördert, und zwar in dem Glauben, für jedes Problem das maßgefertigte Medikament entwickeln zu können. Um herausragende universitäre Positionen zu erhalten, zählt primär die Summe der mit Publikationen gesammelten Impactfaktoren. Ein großer Impactfactor ist im Grunde nur mit molekularen Untersuchungen zu erreichen, hier zählt weniger die medizinsch-klinische Erfahrung. Außerhalb der Universitäten findet durch die Ökonomisierung der Medizin kaum noch klinische Forschung statt. Das alleinige Maß in den Kliniken ist der kumulative Schweregrad der Bewertung von Diagnosebezogenen Fallgruppen (DRGs). Zudem gibt es dort kaum noch ärztliche Erfahrung mit

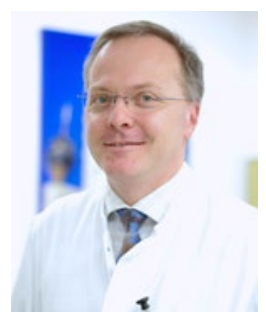

Prof. Dr. med. Stephan Martin

Chefarzt für Diabetologie und Direktor des

Westdeutsches Diabetes- und

Gesundheitszentrum,

Verbund Katholischer Kliniken Düsseldorf

Hohensandweg 37, 40591 Düsseldorf

stephan.martin@vkkd-kliniken.de

Diabetes mellitus, sodass sich die Frage nach klinisch-diabetologischer Forschung meist gar nicht mehr stellt.

Schon Innovationen aus den 1990ern kaum bezahlbar Typ-2-Diabetes entsteht durch die Lebensweise. Logisch wäre daher, Programme zur Bekämpfung der Ursachen zu entwickeln. Dabei sollte nicht nur der Prädiabetes fokussiert werden, sondern auch der manifeste Typ-2-Diabetes, der durchaus in Remission gehen kann. Um im Bild der Kriegsführung zu bleiben: Wir brauchen Waffen, die wirklich den Feind treffen und nicht nur für Rauch und Lärm sorgen. Warum werden weltweit Milliarden öffentlicher Gelder in die molekulare Diabetesforschung investiert, wenn sich die Gesundheitspolitik schon heute nicht mehr fähig sieht, die Innovationen von vor 20 Jahren zu bezahlen? Zuletzt wurden viele innovative Diabetespräparate, deren Wirkprinzip in den 1990er Jahren erstmals beschrieben wurde, aus Kostengründen vom deutschen Markt verbannt. Angenommen die heutige molekulare Forschung identifiziert wirklich ein wesentliches Target, das ungünstigen Lebensstil kompensieren könnte, würden wir das Medikament, das frühestens in 20 Jahren im Handel wäre, überhaupt bezahlen können? Denn dann haben wir noch weniger Beitragszahler und noch mehr Bedürftige. Zudem würde schnell das Sozialgesetzbuch gezückt, wonach das öffentliche Gesundheitswesen keine Lifestyle-Medikamente bezahlen darf.

\section{Investion in Versorgungsforschung wichtig}

Aktuell gewinnt die Diabetologie vielleicht die eine oder andere Schlacht, der Krieg gegen Typ-2-Diabetes ist verloren. Wie bei der globalen Erwärmung können wir maximal noch die Effekte mildern. Wichtig wäre es, die Versorgungsforschung zu stärken, indem die Gelder aus dem molekularen Bereich umgeleitet werden. Doch es gibt keine Lobbyisten dafür. Und auch wenn die Politik reagieren würde, gäbe es wohl auch nicht genug Experten, die die Gelder zielgerichtet dafür einsetzen könnten. Im Krieg gegen den Diabetes sind uns halt die Bodentruppen abhanden gekommen, die bereit sind, in der ersten Reihe zu kämpfen. 